\title{
ASSINATURA DA DEPOSIÇÃO ATMOSFÉRICA DE TESTES NUCLEARES EM SEDIMENTOS DA COSTA BRASILEIRA $\left({ }^{240+239} \mathrm{Pu} \mathrm{E}^{137} \mathrm{Cs}\right)$
}

Christian J. Sanders*, Sambasiva R. Patchineelam, Wilson Machado, Ana Luiza S. Albuquerque e Emmanoel V. Silva-Filho Departamento de Geoquímica, Universidade Federal Fluminense, Outeiro S. João Batista, s/n, 24020-141 Niterói - RJ, Brasil Pedro P. Caldeira

Departamento de Biofísica, Instituto de Biofísica Carlos Chagas Filho, Universidade Federal do Rio de Janeiro, Av. Carlos Chagas Filho, s/n, 21941-902 Rio de Janeiro - RJ, Brasil

Luciana M. Sanders

Instituto de Biologia, Universidade Federal do Rio de Janeiro, Ilha do Fundão, Cidade Universitária, 21941-971 Rio de Janeiro - RJ, Brasil

Recebido em 13/10/11; aceito em 6/1/12; publicado na web em 30/4/12

\begin{abstract}
NUCLEAR TEST FALLOUT SIGNATURES IN SEDIMENTS ON THE BRAZILILIAN COAST $\left({ }^{240+239} \mathrm{Pu}\right.$ AND $\left.{ }^{137} \mathrm{Cs}\right)$. The aim of this review is to take a look at Cold War era nuclear tests signatures found in Brazilian coastal sediments. Both ${ }^{137} \mathrm{Cs}$ and ${ }^{240+239} \mathrm{Pu}$ signatures have been documented in mangrove, coastal mudflats and continental shelf sediments, associated with above ground nuclear tests beginning in the 1950 's. The dates associated to the anthropogenic radionuclide signatures ${ }^{137} \mathrm{Cs}$ and ${ }^{240+239} \mathrm{Pu}$ along sediment columns are confirmed by ${ }^{210} \mathrm{~Pb}$ geochronology in many of the studies highlighted in this review. The results outlined in this review characterize the extent to which nuclear fallout products reach the Brazilian coast in quantities sufficient for detection, allowing the use of these radioisotopes as geochronometers.
\end{abstract}

Keywords : ${ }^{137} \mathrm{Cs} ;{ }^{240+239} \mathrm{Pu}$; geochronology.

\section{INTRODUÇÃO}

Durante o período da guerra fria, testes nucleares eram realizados rotineiramente. Produtos da fissão nuclear (como os radioisótopos de $\mathrm{Pu}$ e ${ }^{137} \mathrm{Cs}$ ) dos testes realizados pelos EUA e União Soviética, entre outros (Figura 1), foram depositados globalmente entre as décadas de 1950 e 1960. Um pico de atividade em 1963/64 pode ser reconhecido em todo o mundo como um marcador geoestratigráfico em testemunhos de sedimentos, gelo e turfa. Entretanto, o fallout estratosférico dos testes nucleares não foi bem estudado no Hemisfério Sul, devido em parte aos menores inventários dos radionuclídeos antrópicos vindos destes testes, originados principalmente do Hemisfério Norte. ${ }^{1}$
Diversos estudos avaliaram as atividades de ${ }^{137} \mathrm{Cs}$ e/ou $\mathrm{Pu}$ em sedimentos de diferentes áreas costeiras no Brasil , ${ }^{2-13}$ incluindo uma revisão geral sobre radionuclídeos naturais e antrópicos no Brasil. ${ }^{5} \mathrm{~A}$ aplicação destes radiotraçadores em estudos de dinâmica sedimentar merece especial atenção, pois ambos podem ser decisivos para uma maior confiabilidade na datação de sedimentos. Existe uma maior escassez de estudos no Brasil examinando a deposição de ${ }^{240+239} \mathrm{Pu}$ para identificar a atividade específica do fallout nuclear, ${ }^{6}$ embora o uso do $\mathrm{Pu}$ pareça oferecer mais vantagens do que o uso do ${ }^{137} \mathrm{Cs}$ na confirmação de datações, por exemplo, devido ao Pu ser relativamente mais imóvel em sedimentos de águas salinas. ${ }^{14}$ Por outro lado, o uso do ${ }^{137} \mathrm{Cs}$ tem sido bem sucedido em diversos ambientes, incluindo

\section{Testes nucleares atmosféricos}

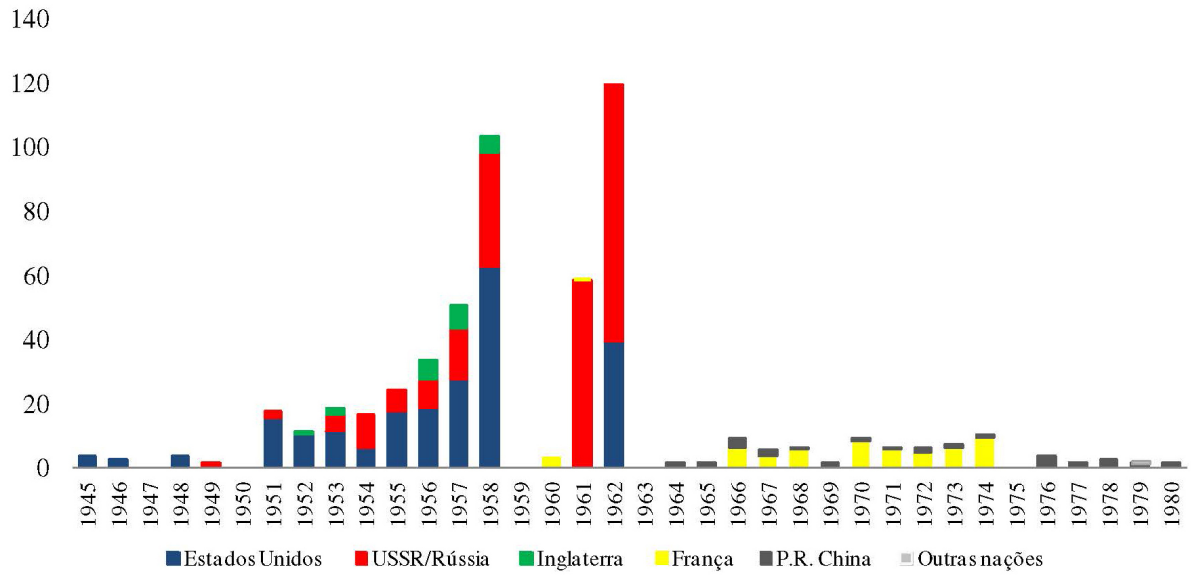

Figura 1. Quantidade e origem de testes nucleares atmosféricos. Dados obtidos em http://www.ctbto.org/fileadmin/user_upload/pdf/Sipri_table12b.pdf e referências nele compiladas 
Tabela 1. Atividades detectadas de ${ }^{239+240} \mathrm{Pu}$ em sedimentos costeiros brasileiros

\begin{tabular}{|c|c|c|c|}
\hline Área de estudo & ${ }^{239+240} \mathrm{Pu}\left(\mathrm{Bq} \mathrm{kg}^{-1}\right)$ & Método & Ref \\
\hline Manguezal de Paraty, RJ a & $<0,02$ a 0,19 & ICP-MS & 6 \\
\hline Costa sul e costa sudeste ${ }^{b}$ & 0,015 a 0,15 & Espectrometria de partículas alfa & 11 \\
\hline Costa sul ${ }^{b}$ & 0,044 a 0,228 & Espectrometria de partículas alfa & 12 \\
\hline Costa das regiões norte, nordeste, sudeste e sul ${ }^{\mathrm{b}}$ & 0,03 a 0,18 & Espectrometria de partículas alfa & 10 \\
\hline
\end{tabular}

${ }^{\mathrm{a}}$ testemunho. ${ }^{\mathrm{b}}$ sedimentos superficiais da plataforma continental.

casos nos quais o ${ }^{210} \mathrm{~Pb}$ não pode ser empregado, ${ }^{13}$ apresentando a vantagem de não requerer pré-tratamento (extração química) para sua determinação, em contraste com os isótopos de $\mathrm{Pu}$.

Neste contexto, o presente estudo consiste numa revisão da literatura sobre a determinação destes radionuclídeos em sedimentos costeiros no Brasil. Foram identificadas as possíveis limitações e vantagens do seu uso para a caracterização da geocronologia sedimentar, incluindo o comportamento geoquímico dos radionuclídeos considerados.

\section{ATIVIDADES RELATADAS PARA A COSTA BRASILEIRA}

As determinações das atividades de ${ }^{239+240} \mathrm{Pu}$ em sedimentos marinhos no Brasil foram conduzidas utilizando espectrometria de partículas alfa e, mais recentemente, empregando o inductively coupled plasma mass spectroscopy (ICP-MS) (Tabela 1). Os procedimentos químicos para o uso da técnica por ICP-MS em sedimentos de áreas com baixa atividade estão bem descritos por Ketterer et al. ${ }^{15}$ Para a espectrometria de partículas alfa, foi utilizada eletrodeposição do plutônio em placa de aço polida, após uma série de tratamentos químicos bem descritos por Figueira et al.. ${ }^{2}$

As atividades máximas encontradas nos sedimentos ao longo da costa do Brasil foram caracterizadas por uma baixa variabilidade, considerando-se que estes ambientes variaram de áreas entre marés até a plataforma continental, ocorrendo atividades entre 0,12 e $0,23 \mathrm{~Bq} \mathrm{~kg}^{-1}$ (Tabela 1). Os estudos encontrados para a costa brasileira foram predominantemente dedicados à avaliação de isótopos de $\mathrm{Pu}$ para a caracterização de sua dispersão como contaminantes antrópicos. ${ }^{10-12} \mathrm{O}{ }^{239+240} \mathrm{Pu}$ foi empregado com sucesso para determinar a taxa de sedimentação numa área de manguezal do sudeste brasileiro, validando seu uso para a geocronologia por meio de comparação com a taxa de sedimentação obtida com o emprego do ${ }^{210} \mathrm{~Pb}{ }^{6}$

As medidas para a determinação das atividades do ${ }^{137} \mathrm{Cs}$ em sedimentos marinhos no Brasil foram realizadas com o uso de detectores de radiação gama (fotopico de 661,6 keV), como consta na Tabela 2. De acordo com os estudos de ${ }^{137} \mathrm{Cs}$ em outras regiões do mundo, a atividade máxima é definida em 1963, quando os testes atmosféricos de armas nucleares promoveram um pico de atividade que pode ser

Tabela 2. Atividade máximas de ${ }^{137} \mathbf{C s}$ em testemunhos de sedimentos costeiros brasileiros, determinadas através de espectrometria de radiação gama

\begin{tabular}{lcc}
\hline Área de estudo & ${ }^{137}{\mathrm{Cs}\left(\mathrm{Bq} \mathrm{kg}^{-1}\right)}$ & Ref. \\
\hline Ribeira Bay, $\mathrm{RJ}^{\mathrm{a}}$ & $\sim 1$ & 8 \\
${\text { Cananéia-Iguape, } \mathrm{SP}^{\mathrm{a}}}$ & 3,2 & 17 \\
${\text { Santos Estuário, } \mathrm{SP}^{\mathrm{a}}}$ & 2,6 & 13 \\
Guaratuba Bay, PR $^{\mathrm{a}}$ & 3,2 & 4 \\
Costa Brasileira $^{\mathrm{b}}$ & $0,3-1,8$ & 11 \\
Costa Brasileira $^{\mathrm{b}}$ & $0,28-3,8$ & 9 \\
${\text { Cananéia-Iguape, } \mathrm{SP}^{\mathrm{a}}}_{\text {Costa Brasileira }^{\mathrm{b}}}$ & 6,1 & 3 \\
\end{tabular}

${ }^{\mathrm{a}}$ pico de atividade em testemunho, correspondendo a 1963. ${ }^{\mathrm{b}}$ variação em sedimentos superficiais de plataforma continental. encontrado em escala mundial. ${ }^{16,17}$ De acordo com os estudos no Brasil, as atividade máximas encontrados nos diferentes ambientes sedimentares foram muito mais variáveis do que observado para o $\mathrm{Pu}$, ocorrendo valores entre aproximadamente 1 e $6,1 \mathrm{~Bq} \mathrm{~kg}^{-1}$ (Tabela 2), que puderam ser empregadas como suporte para a estimativa de taxas de sedimentação, ${ }^{3,4,6,8}$ ou até sendo o ${ }^{137} \mathrm{Cs}$ o único radionuclídeo no qual esta estimativa se baseou, ${ }^{13}$ no sistema estuarino de SantosCubatão (SP), no qual as atividades de ${ }^{210} \mathrm{~Pb}$ sofrem influência de fontes industriais, gerando a necessidade do emprego de outros métodos. ${ }^{18,19}$ Uma frequência de aplicação do ${ }^{137} \mathrm{Cs}$ relativamente baixa foi encontrada, mas, mesmo assim, foi bastante superior à quase ausência de aplicação do ${ }^{239+240} \mathrm{Pu}$ para estudos de geocronologia.

\section{COMPORTAMENTO E APLICABILIDADE DOS RADIONUCLÍDEOS}

\section{Plutônio}

O Pu é um importante marcador ambiental e possui seis isótopos principais: ${ }^{238} \mathrm{Pu}\left(\mathrm{t}_{1 / 2}=87,74\right.$ anos $),{ }^{239} \mathrm{Pu}\left(\mathrm{t}_{1 / 2}=241,10\right.$ anos $),{ }^{240} \mathrm{Pu}$ $\left(\mathrm{t}_{1 / 2}=6,561\right.$ anos $),{ }^{241} \mathrm{Pu}\left(\mathrm{t}_{1 / 2}=14,29\right.$ anos $),{ }^{242} \mathrm{Pu}\left(\mathrm{t}_{1 / 2}=373.000\right.$ anos $)$ $\mathrm{e}^{244} \mathrm{Pu}\left(\mathrm{t}_{1 / 2}=8,3 \times 10^{7}\right.$ anos). Os isótopos ${ }^{238} \mathrm{Pu},{ }^{239} \mathrm{Pu},{ }^{240} \mathrm{Pu},{ }^{242} \mathrm{Pu}$ e ${ }^{244} \mathrm{Pu}$ decaem por emissão de partículas $\alpha$, enquanto o ${ }^{241} \mathrm{Pu}$ decai por emissão $\beta$ produzindo ${ }^{241} \mathrm{Am}\left(\mathrm{t}_{1 / 2}=432,6\right.$ anos $) .{ }^{20}$ Processos de redução-oxidação são vitais para a determinação do comportamento ambiental do Pu. Este elemento possui comportamentos análogos para misturas onde prevalecem os estados +4 e +5 e, sob condições extremamente redutoras, também pode existir em seu estado de oxidação +3. O Pu é menos móvel e, portanto, mais fortemente associado com os sedimentos quando presente em seu estado reduzido +3 . Em geral, este elemento possui fases sólidas diferentes em águas doces e ambientes marinhos, o que acarreta em seu uso eficiente como traçador de transporte de partículas no ambiente. Além das ocorrências naturais do ${ }^{239} \mathrm{Pu}$ na superfície da terra mencionadas, os radioisótopos de Pu estão largamente distribuídos no ambiente como resultado da produção antrópica nas últimas 6 décadas. O modo de produção é, novamente, a captura de nêutrons em uma bomba de fissão nuclear, ou em um reator para produção elétrica, ou para $\operatorname{transmutar}{ }^{238} \mathrm{U}$ em ${ }^{239} \mathrm{Pu}$ para armamentos. Como resultado, essencialmente todo o ${ }^{239} \mathrm{Pu}$ na superfície da Terra é de origem antrópica.

As maiores fontes de fallout global de Pu foram os testes nucleares, particularmente na atmosfera. Uma vez na troposfera, o Pu se associa às partículas do aerossol e é removido da atmosfera por deposição úmida e/ou seca. Nos solos, o Pu se associa com fases específicas, como óxidos de ferro/manganês e ácidos húmicos. Além dos testes de armas, outra fonte importante inclui acidentes nucleares como o de Chernobyl (1986), fábricas de reprocessamento de combustível nuclear (e.g., Sellafield, UK), desintegração atmosféricas de geradores elétricos de satélites (acidente SNAP em 1964) e emissões de fábricas voltadas para manutenção e produção de componentes de armas.

A deposição atmosférica de $\mathrm{Pu}$, associada a produtos de fissão como o ${ }^{137} \mathrm{Cs}\left(\mathrm{t}_{1 / 2}=30,07\right.$ anos), apresenta um pico pronunciado em 1963, seguido de uma aguda diminuição na deposição sedimentar 
(Figura 2). Esta aguda diminuição coincide com o tratado de banimento de testes nucleares assinados por EUA, União Soviética e Reino Unido. O tempo de residência na atmosfera para radionuclídeos introduzidos na troposfera é tipicamente menor do que 1 mês e os inventários acumulados de fallout variam amplamente em relação ao posicionamento global. ${ }^{16}$ Os inventários mais altos são encontrados no Hemisfério Norte em latitudes medianas, enquanto que os inventários menores ocorrem conforme nos aproximamos dos Polos e do Equador.

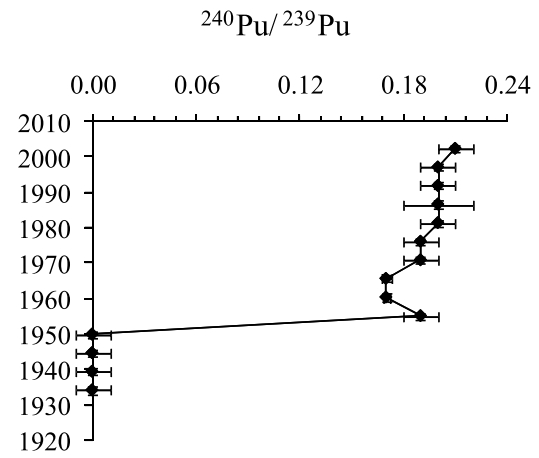

Figura 2. Razão atômica do ${ }^{240} \mathrm{Pu}{ }^{239} \mathrm{Pu}$ ao longo de um perfil sedimentar de área de manguezal em Paraty, $R^{6}$

Os menores valores de fallout total ocorrem no Hemisfério Sul, por conta do menor número de testes de aparatos nucleares. Pelo fato ainda da baixa mistura entre massas de ar estratosféricos entre hemisférios, ${ }^{16}$ inventários de radionuclídeos advindos de fallout são até 5 vezes maiores no Hemisfério Norte. Apesar da dependência em relação à latitude, os inventários deposicionais também exibem variações resultantes do clima, com a precipitação atmosférica como principal mecanismo de remoção do fallout. Para latitudes equivalentes, valores maiores de inventários de fallout são observados em climas com maior média anual de precipitações, consequentemente, locais com climas marinhos possuem inventários maiores do que desertos ou padrões de clima úmidos continentais. ${ }^{21}$ Existem pequenas diferenças na composição isotópica entre os hemisférios. Levando em consideração que o Pu é sintético em sua origem, sua composição isotópica varia amplamente de acordo com seu mecanismo de produção; dessa forma, a variância intrínseca serve como base para uma grande variedade de possibilidades de uso como traçador de ambientes atmosférico e marinho. A razão atômica ${ }^{240} \mathrm{Pu} /{ }^{239} \mathrm{Pu}$ fornece sinais no sedimento que podem ser usados para identificar as fontes e o tempo associado à sua exposição ambiental. ${ }^{22}$

\section{Césio}

$\mathrm{O}{ }^{137} \mathrm{Cs}$ é depositado por fallout estratosférico com um histórico bem definido e um sinal reconhecível de maneira global. Datações geoestratigráficas determinam um ou mais eventos associados a deposições em camadas associados a datas especificas. ${ }^{1-7}$ Em um padrão deposicional ideal, a acumulação por fallout seria precisamente coincidente com o histórico de deposição, entretanto, em sistemas reais, o fallout de nuclideos aparece em perfis de sedimento como uma mistura da deposição atmosférica direta com o fallout já previamente depositado, transportado de outras regiões. Estes processos de mistura, junto com a média de tempo que o material permanece em suspensão, podem ter um efeito amortecedor na precisão dos padrões de deposição. Como resultado, normalmente só pode especificar o começo do fallout (1952-1954) e a atividade máxima (1963). Na maioria dos casos, os picos de pré-moratória e pós-moratória não estão devidamente separados e, frequentemente, distúrbios no testemunho adicionam perda de resolução e distorção na datação. Misturas pós-deposicionais devido a processos físicos ou bioturbações podem introduzir ambiguidade na medida da data de começo do fallout. Entretanto, na maioria dos casos, o pico de atividade de 1963 continua distinto. Dessa maneira, a análise da relação qualidade/integridade de um testemunho não perturbado pode ser definida através de análises ou modelagem da atividade do perfil contra o tempo de deposição da fonte atmosférica. Testemunhos exibindo perfis de atividade erráticos tendem a ter utilidade limitada em estudos onde é necessária uma linha do tempo acurada para o estudo de outros fenômenos, como históricos climáticos ou de poluição. ${ }^{4}$

Mesmo se podendo utilizar uma geocronologia por ${ }^{210} \mathrm{~Pb}$ em ambientes marinhos, é muito recomendável validar os resultados empregando um segundo traçador independente, que tem sido preferencialmente o ${ }^{137} \mathrm{Cs}$, como refletido por este trabalho para a costa brasileira. Levando em conta os cuidados indicados, as cronoestratigrafias de ${ }^{137} \mathrm{Cs}$ são frequentemente bem sucedidas, mesmo sabendo-se que uma perda considerável de atividade ocorreu devido ao seu decaimento (em torno de dois terços do inventário do fallout de ${ }^{137}$ Cs originalmente depositado terá decaído até o ano de 2010). Esta limitação pode ser compensada com um maior investimento no tempo (horas/dias) de contagem da emissão gama.

$\mathrm{O}$ coeficiente de sorção, Kd, é um parâmetro operacional comumente utilizado para descrever o particionamento de um elemento entre sólido-adsorvido e fases de solução $(\mathrm{Kd}=$ Csolid/Csolution $)$ com a unidade de mL/g. Assim, um alto valor de Kd é um forte indicativo da tendência de associação de partículas e, por consequência, sua imobilidade relativa como um solvente real, enquanto baixos valores de Kd indicam solubilidade significativa e tendência da substância de ser transportada em forma de solução. Em Kd determinados, são limitadas as circunstâncias ambientais nas quais foi medido e pode, obviamente, ser afetado gravemente por fatores como estado de oxidação/especiação do elemento, temperatura ambiental, características da fase aquosa ( $\mathrm{pH}$, força iônica, e presença de complexantes) e propriedades físicas e químicas da fase sólida (como granulometria, composição mineral, entre outros). Em água salgada o Kd para o Cs é muito menor do que em sistemas de água doce, devido à competição entre Cs e grandes concentrações de outros cátions alcalinos. Isto confere uma maior mobilidade, que pode alterar o seu registro sedimentar original, o que deve ser também avaliado (Figura 3).

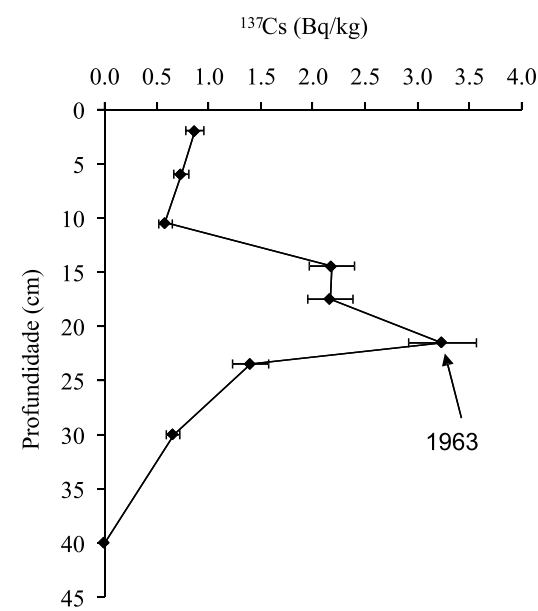

Figura 3. Perfil de ${ }^{137} \mathrm{Cs}$ em um testemunho estuarino da Baía de Guaratuba $(P R)$, com um pico de atividade que corresponde a $1963^{4}$

\section{CONCLUSÃO}

A detecção de perfis reconhecíveis de ${ }^{137} \mathrm{Cs}$ e ${ }^{239+240} \mathrm{Pu}$ em ambientes costeiros do Brasil é promissora para se determinar geocronologias 
sedimentares a partir da década de 1950. Existe uma escassez de dados sobre radinuclídeos relacionados a testes de aparatos nucleares como ${ }^{239+240} \mathrm{Pu}$ e ${ }^{137} \mathrm{Cs}$ no Hemisfério Sul, em parte decorrente dos inventários de fallout $\sim 5$ vezes mais baixos em relação aos do Hemisfério Norte.

Os estudos no Brasil, compilados nesta revisão, demonstram que ambos os registros vindos do fallout nuclear podem ser aplicados com sucesso como traçadores geocronológicos, como algumas vezes confirmado com o emprego concomitante do ${ }^{210} \mathrm{~Pb}$. Enquanto o ${ }^{137} \mathrm{Cs}$ possui a vantagem de não carecer de pré-tratamento laborioso para a sua determinação, os isótopos de $\mathrm{Pu}$ apresentam um potencial particular para estudos geocronológicos, pois não decaíram significativamente nos últimos 50 anos, possuem menor mobilidade e permitem a confirmação da fonte por meio de razões isotópicas.

\section{AGRADECIMENTOS}

À CAPES/FAPERJ, pela bolsa concedida para C. J. Sanders (Proc. E 26/101.952/2009).

\section{REFERÊNCIAS}

1. Kelley, J. M.; Bond, L. A.; Beasley, T. M.; Sci. Total Environ. 1999, 237/238, 483 .

2. Figueira, R. C. L.; Cunha, I. I. L.; Quim. Nova 1998, 21, 73.

3. Saito, R. T.; Figueira, R. C. L.; Tesslier, M. G.; Cunha, I. I. L.; J. Radioanal. Nucl. Chem. 2001, 250, 109.

4. Sanders, C. J.; Santos, I. R.; Silva, E. V.; Patchineelam, S. R.; Mar. Pollut. Bull. 2006, 52, 1089.

5. Santos, I. R.; Burnett, W. C.; Godoy, J. M.; Braz. J. Oceanogr. 2008, 56, 115.

6. Sanders, C. J.; Smoak, J. M.; Sanders, L. M.; Waters, M. N.; Patchineelam, S. R.; Ketterer, M. E.; J. Radioanal. Nucl. Chem. 2010, 283, 593.
7. Patchnieelam, S. M.; Sanders, C. J.; Smoak, J. M.; Zem, R. C.; Oliveira, G.; Patchineelam S. R.; J. Braz. Chem. Soc. 2011, 22, 120.

8. Gomes, C. F.; Godoy, J. M.; Godoy, M. L. D. P.; Carvalho, Z. L.; Lopes, R. T.; Sanchez-Cabeza, J.; Osvath, I.; Lacerda, L. D.; J. Environ. Radioact. 2011, 102, 871.

9. Godoy, J. M.; Carvalho, Z. L.; Fernandes, F. C.; Danelon, O. M.; Ferreira, A. C. M.; Roldao, L. A.; J. Environ. Radioact. 2003, 70, 193.

10. Cunha, I.; Figueira, R. C. L.; Saito, R. T.; J. Radioanal. Nucl. Chem. 1999, 239, 3477.

11. Figueira, R. C. L.; Tessler, M. G.; de Mahiques, M. M.; Cunha, I. I. L.; Sci. Total Environ. 2006, 357, 146.

12. Figueira, R. C. L.; Furtado, V. V.; Tessler M. G.; Cunha, I. I. L.; Braz. J. Oceanogr. 2003, 51, 55.

13. Martins, C. C.; Bícego, M. C.; Mahiques, M. M.; Figueira, R. C. L.; Tessler, M. G.; Montone, R. C.; Environ. Pollut. 2010, 158, 3355.

14. Zheng, J.; Yamada, M.; Environ. Sci. Technol. 2004, 38, 3498.

15. Ketterer, M. E.; Hafer, K. M.; Jones, V. J.; Appleby, P. G.; Sci. Total Environ. 2004, 322, 221.

16. Bennett, B. G.; Health Phys. 2002, 1456, 644.

17. Sanders, C. J.; Smoak, J. M.; Sathy, M.; Araripe, D. E.; Sanders, L. M.; Patchineelam, S. R.; Environ. Earth Sci. 2010, 60, 1291.

18. Machado, W.; Luiz-Silva, W.; Sanders, C. J.; Patchineelam, S. R.; J. Environ. Radioact. 2008, 99, 1329.

19. Luiz-Silva, W.; Machado, W.; Matos, R. H. R.; J. Braz. Chem. Soc. 2008, 19, 1490.

20. Taylor, D. M. Em Environmental plutonium - creation of the universe to twenty-first century mankind; Taylor, D. M.; Kudo, A., eds.; Elsevier Science: Amsterdam, 2001.

21. Beck, H. L.; Bennett, B. G.; Health Phys. 2002, 82, 591

22. Ketterer, M. E. Em Applications of Transuranics as Tracers and Chronometers in the Environment; Ketterer, M. E.; Zheng, J.; Yamada, M., eds.; Baskaran M: New York, 2011, cap. 20. 